\title{
Effect of High Pressure Carbon Dioxide Exposure on the Permeation Properties of Asymmetric Polysulfone Membranes for Gas Separation
}

\author{
A. F. Ismail ${ }^{1 * *}$, L. Wong ${ }^{2}$ \& T. Matsuura ${ }^{3}$ \\ ${ }^{1 \& 2}$ Membrane Research Unit, Facklty of Chemical \& Natural Resources Engineering, Universiti of Teknologi \\ Malaysia, 81310 Skudai, Johor Darul Takzim, Malaysia \\ ${ }^{3}$ Industrial Membrane Research Institute, Department of Chemical Engineering, University of Ottawa, Ottawa, \\ Ontario, Canada K1N $6 \mathrm{~N} 5$
}

\begin{abstract}
In this study, the dependence of permeability on the feed pressure for glassy asymmetric polysulfone membranes has been investigated. Pure $\mathrm{CO}_{2}$ and $\mathrm{CH}_{4}$ were used as test gases. In pure gas experiments with $\mathrm{CO}_{2}$, the untreated membrane showed that the carbon dioxide permeation rate increased with increasing feed pressure from one bar onwards. This is because carbon dioxide behaves as a plasticizing agent by expanding the polymer lattice and jncreased the frequency of molecular motions of the polymer. Moreover, high carbon dioxide concentration in the polymer film disrupts the chain packing, thereby leading to a larger free volume and enhanced rates of segmental motions. Due to swelling of the polymer matrix, the structure of the polymer matrix is loosened and consequently polymer chains become more flexible. For the treated membrane, the permeability of carbon dioxide does not increase with feed pressure but gives a steady state value over the feed pressure ranges investigated. This was in agreement with scanning electron microscopy studies, which revealed that heat treatment densified the membrane skin layer. Thus, a subsequent heat treatment to the membrane is necessary in suppressing the plasticization effect.
\end{abstract}

Keyzords: Carbon dioxide, plasticization, gas separation

\subsection{INTRODUCTION}

Gas separation using integrally skinned asymmetric membranes made from glassy polymers is a proven technology that has found a wide range of industrial applications [1]. These membranes as illustrated in Figure 1 consist of an extremely thin and dense surface layer $(0.1 \mu \mathrm{m}$ to $1 \mu \mathrm{m})$ and a much thicker porous sub-layer (100 $\mu \mathrm{m}$ to 200 ․m) of the same material, on which the dense layer is supported. The skin layer performs the separation with a high flux because of its high density and thinness. The porous sublayer provides the mechanical strength while the gases may permeate

\footnotetext{
" Corresponding to: A. F. Ismail (emanl: afauzi@utm.my)
}

through it with little resistance [2]. However, problems arise due to plasticization of glassy polymer [3]. This phenomenon if related to the dilation in the polymeric matrix induced by the

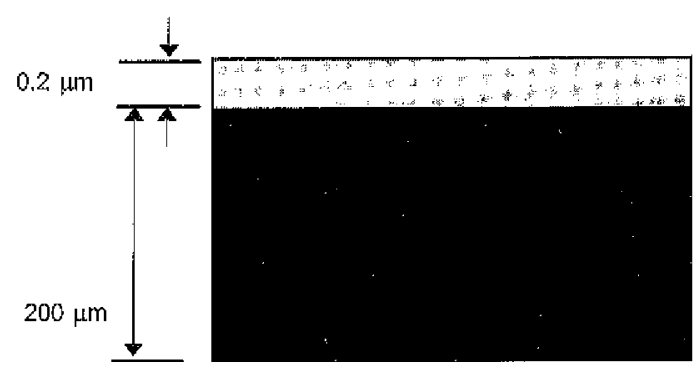

Figure 1 A schematic illustration of the structure of integrally-skinned asymmetric membranes 
highly sorbed penetrant molecules. This becomes larger as the penetrant concentration increases, which further allows greater mobility of the chain segments and higher diffusion rates [4]. Consequently, membrane plasticization flexibilizes the discriminating matrix with a reduction in the permselectivity of the membrane at sufficiently high driving pressure. In other words, plasticization may be interpreted as a weakening effect on the polymer, which eventually results in the swollen materials unsuitable for gas separation applications. Therefore, understanding of plasticization phenomenon is essential especially at high pressure condition in order to develop high performance glassy polymer membrane $[5,6]$.

Efforts have therefore been made to improve the separation performance of asymmetric membranes through either phase inversion process optimization and/or post treatment methods [6].

In this paper the effect of exposure of asymmetric polysulfone membranes to high pressure $\mathrm{CO}_{2}$ on their gas permeation properties is thoroughly studied since through the best of our knowledge only few studies have been made for this particular case. Another novel aspect of this study is the study of the the effect of the heattreatment polysulfone membranes on their stability in gas separation performance.

\subsection{EXPERIMENTAL}

\subsection{Membrane Preparation}

Polysulfone (PSF) manufactured by Amoco Chemical (Europe) S.A. under the trade name "Udel polysulfone P-1700" was selected as the polymer for this study primarily because of its low cost and widespread use as a commercial membrane material for gas separation. Although the polysulfone have a reasonably high $T_{g}$, the polymers are significantly plasticized by highly sorbing penetrants at high pressure [7].

Asymmetric flat sheet membranes were prepared by the dry/wet phase inversion process from casting solutions composed of polysulfone, $\mathrm{N}, \mathrm{N}$ '- dimethylacetamide (less volatile solvent), tetrahydrofuran (more volatile solvent) and ethanol (nonsolvent). The polymer solution was cast on a clean glass plate using a pneumatically controlled flat sheet membrane casting system constructed in our laboratory. Forced convective evaporation was induced on the cast polymer solution film before it was immersed in a water bath overnight at room temperature. The membrane was further transfered to a methanol bath where the membrane was kept for two hour for solvent exchange and then airdried at least for one day at room temperature. Some membranes were further heat-treated by placing them in a forced hot air oven at a predetermined temperature.

Before testing, the membrane was coated with a highly permeable elastomeric silicone polymer (polydimethylsiloxane) (Slygard 184, Dow Corning). The top skin side of the membrane was kept in contact with a 3\% w/w silicone solution in $\mathrm{n}$-hexane for 3 minutes. Then, the membrane was placed in an oven at $60^{\circ} \mathrm{C}$ for $2 \mathrm{hrs}$ to allow curing of the silicone layer. The coating is a standard method, which can repair any imperfections in the active layer, and will not alter the permeation characteristics of the asymmetric membrane material (Figure 2). Thus, coating allows the membrane to exhibit permeation properties close to the inherent characteristics of the membrane polymer itself $[8,9]$.

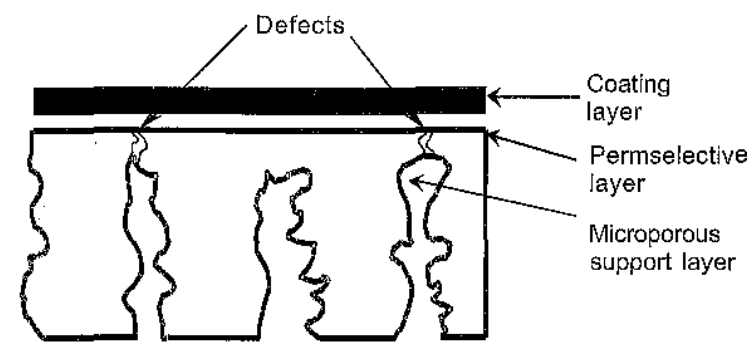

Figure 2 Schematic of coated gas-separation membranes

\subsection{Gas Permeation Experiments}

The high pressure permeation setup is a constant pressure system as illustrated schematically in Figure 3. The gas permeation tests were performed using a permeation cell. The permeation cell was constructed using stainless steel 316 , allowed the 


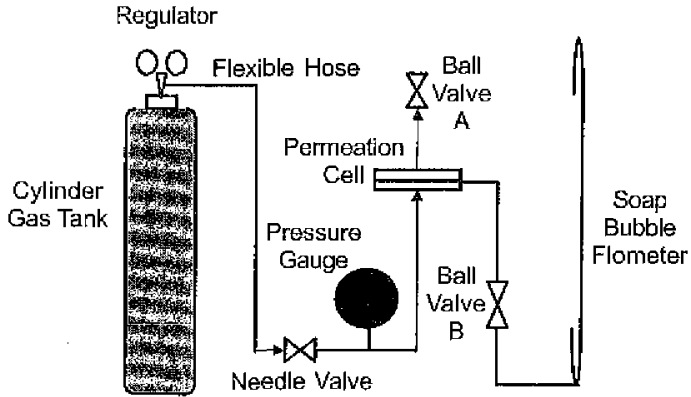

Figure 3 Pre gas permeation testing system

permeation tests at elevated pressures (up to 13 bars). In the permeation cell, the membrane samples were placed on a Belkipor ST-CL3 filter medium that is adurable and pleatab le $316 \mathrm{~L}$ stainless steel medium sintered into a graded pore structure. The volumetric gas flow rates were determined using a soap bubble flow meter reading to $0.05 \mathrm{~cm}^{3}$, attached to the permeation cell with a thick-walled rubber hose or tubing. The effective membrane area was $13.5 \mathrm{~cm}^{2}$. All permeation experiments were single gas experiments performed at $25 \pm 2^{\circ} \mathrm{C}$.

The permeance, $P / l$, and was calculated by

$$
\left(\frac{P}{l}\right)_{i}=\frac{Q_{10^{6}}}{\left(\Delta_{p}\right)(A)}
$$

where $Q_{i}$ is the volumetric flow rate of gas ' $i$ ' at standard temperature and pressure $\left(\mathrm{cm}^{3} / \mathrm{s}\right), \Delta_{p}$ is the transmembrane pressure difference $(\mathrm{cmHg})$, and $A$ is the effective area of the membrane $\left(\mathrm{cm}^{2}\right)$. Permances are expressed in gas permeation units, GPU, where

$$
1 \mathrm{GPU}=10^{-6} \mathrm{~cm}^{3}(\mathrm{STP}) \cdot \mathrm{cm}^{-2} \cdot \mathrm{s}^{-1} \cdot \mathrm{cmHg}^{-1}
$$

\subsection{Differential Scanning Calorimetry}

A membrane sample was loaded into a sample pan for thermal analysis with a, differential scanning calorimeter (Perkin-Elmer DSC-7) equipped with a Thermal Analysis Data Station (TADS). The glass transition temperatures obtained from two samples were averaged and reported.

\subsection{Scanning Electron Microscopy}

Membrane morphology was studied by scanning electron microscopy (SEM) technique. The membrane samples were prepared by cryogenic breaking of the membranes, followed by coating with a thin layer of gold.

\subsection{RESULTS AND DISCUSSION}

Figure 4 shows $\mathrm{CH}_{4}$ and $\mathrm{CO}_{2}$ permeance versus feed gas pressure. As stated earlier, the data were obtained from pure gas permeation experiments. From Figure 4, $\mathrm{CH}_{4}$ permeance is almost independent from the feed gas pressure, as documented and adequately explained by the dualmode model. According to Sanders [10], decrease in permeability stems from a stronger decreasing tendency of solubility than increasing tendency of diffusivity. Sanders also mentioned that a 'microvoid-situation' would appear at low levels of penetrant in the polymer, while at higher penetrant concentrations, such microvoids would become saturated, causing the decrease in gas permeability.

From Figure 4, $\mathrm{CO}_{2}$ permeance exhibited an increasing tendency with an increase in the feed pressure. This is very typical for $\mathrm{CO}_{2}$ Permeance through membranes made of glassy-polymers. According to Bos et al. [11], the increase in permeability due to is possible because of an

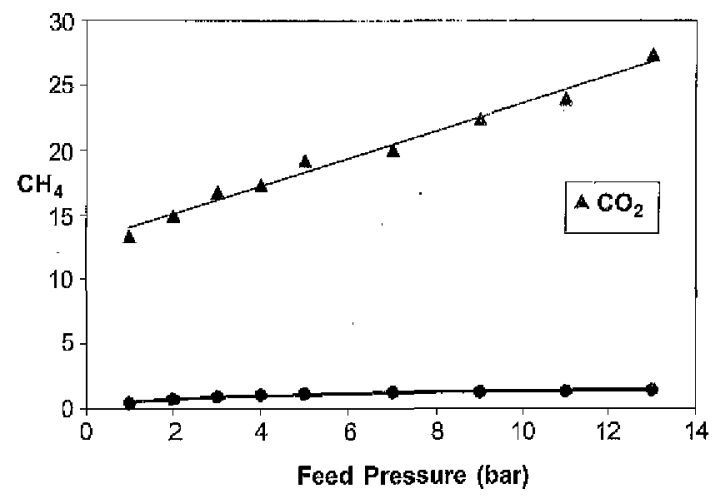

Figure $4 \mathrm{CO}_{2}$ and $\mathrm{CH}_{4}$ permeability of the asymmetric polysulfone as a function of the feed pressures 
increase in chain mobility: A large amount of penetrant in a polymeric membrane disiupts chain packing, leading to increased segmental motions. This phenomenon is well known as plasticization effect.

Another explanation is that the diffusion coefficient in creases with the pressure much more rapidly than the decrease in solubility [10]. Moreover, some researchers [12-14] have found the $\mathrm{CO}_{2}$ sorption results in dilation of polymers, which allows easy passage of the carbon dioxide molecules and influence the mass transport. In other words, the activation energy for diffusion decreases with increasing of penetrant concentration because of the presence of $\mathrm{CO}_{2}$ may loosen the polymer matrix by separation of chain segments for a diffusional jump.

White et al. [14], on the other hand, proposed that the asymmetric membranes prepared by precipitation process possess morphology where small nodules of polymer are tied together with regions of lower density polymer chains. The exposure to highly soluble plasticizer such as $\mathrm{CO}_{2}$ results in the loosely packed chains which are more susceptible to movement and structural change.

Figure 5 shows $\mathrm{CO}_{2}$ permeance versus feed pressure for both heat-treated and untreated membranes. Regarding the data for the untreated membrane, the data in Figure 6 is slightly different from the ones in Figure 5 since different membrane

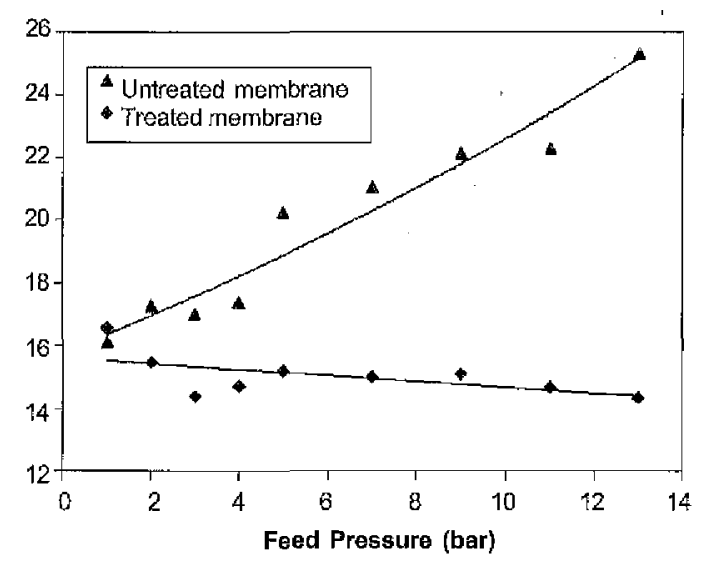

Figure 5 Carbon dioxide permeability of the asymmetric polysulfone as a function of the feed pressures samples were used in permeation experiments. Hence, there is slight difference in the permeance data. Plasticization effect is clearly observed for the untreated membrane. For the heat-treated membrane, the permeance was nearly equal to that of the untreated membrane at $1 \mathrm{bar}$. The decreased with an increase in pressure but the change was signilicantly less than untreated membrane, indicating improvement of performance stability. This is due to better packing of polymer chains and decrease in free volume in the heat-treated membrane. This is also probably due in part to the lower segmental (rotational) mobility, i.e., greater chain stiffness, of the polysulfone. This phenomenon is in agreement with th at reported by Paul and Yampol [16].

Increase in the packing density of the polymer is further supported by the SEM pictures (Figure 6). The figure shows that the size of the pores right underneath the thin skin layer decreased by the heat-treatment. This seems consistent with denser polymer packing and lower free volume in the thin skin layer of the heat-treated membrane. Similar observations have been reported b y Bos et al. [3] and Krol [17]. Thermal treatment induces rearrangements of polymer chains towards a new configurationally state. It was observed that restriction of the polymer chain mobility after heattreatment could impede gas transport [18]. Thus,

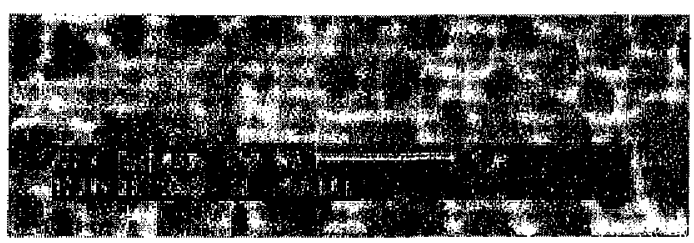

(a)

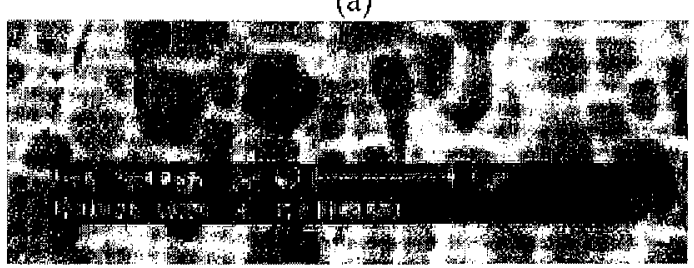

(b)

Figure 6 SEM pictures of (a) before heat treatment and (b) after heat treatment at $140^{\circ} \mathrm{C}$ for polysulfone flat sheet membranes 
heat-treatment method is effective to stabilize the performance of asymmetric membranes.

\subsection{CONCLUSION}

The following conclusions can be drawn from the experimental work in th is study.

(1) The $\mathrm{CH}_{4}$ permeance of the asymmetric polysulfone membrane fabricated by the phase inversion technique followed by silicone rubber coating decreases as the feed $\mathrm{CH}_{4}$ pressure increases in accordance with the dual-sorption behavior of membranes made of glassy polymers.

(2) The $\mathrm{CO}_{2}$ permeance, on the other hand, increases with an increase in the feed $\mathrm{CO}_{2}$ pressure. This is due to much reported plasticization effect.

(3) Heat- treatment of the asymmetric polysulfone membrane suppresses the plasticization effect significantly.

(4) The stabilization by heat-treatment is due to the denser packing of macromolecules and free volume reduction. This is evidenced, at least indirectly, by SEM observation which revealed shrinkage of the pores immediately underneath the dense skin layer.

\section{REFERENCES}

[1] Strathmann, H. 1981. Membrane Separation Processes. J. Membr. Sci. 9: 121.

[2] Baker, R. W. 1991. Membrane Separation System Recent Developments and Future Directions. New Jersey: Noyes Publication.

[3] Bos, A., I. G. M. Punt, M. Wessling, and H. Strathmann. 1998. Plasticization Resistant Glassy Polyimide Membranes for High Pressure $\mathrm{CO}_{2} / \mathrm{CH}_{4}$ Separations. Sep. Purif. Techn. 14: 27.

[4] Puleo, A. C., D. R. Paul, and S. S. Kelley. 1989. The Effect of Degree of Acetylation on Gas Sorption and Transport Behaviour in Cellulose Acetate. J. Membr. Sci. 47: 301.

[5] Ismail, A. F. and W. Lo rna. 2002. Penetrantinduced Plasticization Phenomenon in
Glassy Polymers for Gas Separation Membrane. Sep . Purif. Techn. 27: 173.

[6] Ismail, A. F. and W. Lorna. 2003. Suppression of Plasticization in Polysulfone Membranes for Gas Separation by Heat-treatment Technique. Sep. Purif. Techn. 30: 37.

[7] Fried, J. R., H. C. Liu, and C. Zhang. 1989. Effect of Sorbed Carbon Dioxide on the Dynamic Mechanical Properties of Glassy Polymers. J. Polym. Sci. Part C: Polym. Lett. 27: 385 .

[8] Ismail, A. F., S. J. Shilton, I. R. Dunkin, and S. L. Gallivan. 1997. Direct Measurement of Rheologically Induced Molecular Orientation in Gas Separation Hollow Fiber Membranes and Effects on Selectivity. $J$. Membr. Sci. 126: 133.

[9] Pfromm, P. H., I. Pinnau, and W. J. Koros. 1993. Gas Transport Through Integralasymmetric Membranes: A Comparison to Isotropic Film Transport Properties. J. Appl. Polym. Sci. 48: 2161.

[10] Sanders, E. S. 1998. Penetrant-induced Plasticization and Gas Permeation in Glassy Polymers. J. Membr. Sci. 37: 63.

[11] Bos, A., I. G. M. Punt, M. Wessling, and H. Strathmann. 1999, $\mathrm{CO}_{2}$-induced Plasticization Phenomena in Glassy Polymer. J. Membr. Sci. 155: 67.

[12] Wessling, M., S. Schoeman, Th. Van den Boomgaard, and C. A. Smolders. 1991. Plasticization of Gas Separation Membranes. Gas Sep. Purif. 5: 222.

[13] Sefcik, M. D. 1986. Dilation of Polycarbonate by Carbon Dioxide. J. Po lym. Sci., Part B: Polym. Phys. 24: 935.

[14] Kamiya, Y., T. Hirose, Y. Naito, and K. Mizoguchi. 1998. Sorptive Dilation of Polysulfone and Poly (ethylene terephthalate) Films by High-pressure Carbon Dioxide. J. Polym. Sci., Part B: Polym. Phys. 26: 159.

[15] White, L. S., T. A. Blinka, H. A. Kloczewski, and I-Fan Wang, 1995. Properties of Polyimide Gas Separation Membrane in Natural Gas Streams. J. Membr. Sci. 103: 73.

[16] Paul, D. R., and Yampol' Skii. 1994. Polymeric Gas Separation Membranes. CRC Press. 
[17] Krol, J. J., M. Boerrigter, and G. H. Koops. 2001. Polyimide Hollow Fiber Gas Separation Membranes: Preparation and the Suppression of Plasticization in Propane/ Propylene Environments. J. Membr. Sci. 184: 275.
[18] Dudley, C. N., B. S choberl, G. K. Sturgill, H. W. Beckham and M. E. Rezac. Influence of Crosslinking Technique on the Physical and Transport Properties of Ethynyl-terminated Monomer/polyetherimide Asymmetric Membranes. J. Membrane Sci. 191: 1-11. 